\title{
High yield synthesis of multi-walled carbon nanotubes by catalytic decomposition of ethane over iron supported on alumina catalyst
}

\author{
B. Louis ${ }^{\mathrm{a}, \mathrm{c}}$, G. Gulino ${ }^{\mathrm{b}, \mathrm{c}}$, R. Vieira ${ }^{\mathrm{a}, \mathrm{c}}$, J. Amadou ${ }^{\mathrm{a}, \mathrm{c}}$, Th. Dintzer ${ }^{\mathrm{a}, \mathrm{c}}$, S. Galvagno ${ }^{\mathrm{b}, \mathrm{c}}$, \\ G. Centi ${ }^{\mathrm{b}, \mathrm{c}}$, M. J. Ledoux ${ }^{\mathrm{a}, \mathrm{c}}$, C. Pham-Huu ${ }^{\mathrm{a}, \mathrm{c}}$
}

\author{
a Laboratoire des Matériaux, Surfaces et Procédés pour la Catalyse \\ (UMR 7515 du CNRS), ECPM-ULP, 25, rue Becquerel, F-67087 Strasbourg Cedex 2, France \\ b Dip. Chimica Industriale e Ingegneria dei Materiali, \\ Universita di Messina, Salita Sperone 31, 98166 Messina, Italy \\ c European Laboratory of Catalysis and Surface Science (ELCASS) \\ To whom all correspondence should be addressed \\ E-mail : vieirar@ecpm.u-strasbg.fr \\ Tel. 33390242675 , Fax. 33390242674
}




\begin{abstract}
Ethane was successfully used as an active and efficient carbon source in a large scale synthesis of high quality carbon nanotubes by chemical vapour deposition (CVD) over $\mathrm{Fe} / \mathrm{Al}_{2} \mathrm{O}_{3}$ catalyst with an iron loading of $20 \mathrm{wt} . \%$. The MWNT yield approaching $50 \mathrm{~g}$ per gram of catalyst per hour at $640{ }^{\circ} \mathrm{C}$ which was among the highest yield reported today in the open literature. The active phase for growing carbon nanotubes is a mixture of a metastable iron carbide $\left(\mathrm{Fe}_{3} \mathrm{C}\right)$ and $\alpha-\mathrm{Fe}$ which were formed in situ by the ethane dissociation followed by carbon diffusion through the starting $\alpha$-Fe phase. The reaction products only contained multi-walled carbon nanotubes with very homogeneous diameters of around $30 \mathrm{~nm}$ and lengths up to several hundred nanometers without any trace of other impurities such as nanoparticles or amorphous soot. According to the observed results ethane is an active carbon source for growing MWNTs with high yield and selectivity. The reaction temperature should be keep $\leq 750{ }^{\circ} \mathrm{C}$ as synthesis carried out at $800{ }^{\circ} \mathrm{C}$ under similar reaction conditions led to the formation of amorphous soot and carbon nanoparticles mixed with nanotubes.
\end{abstract}




\section{Introduction}

Carbon nanofilaments, either nanotubes or nanofibers, have received increasing scientific and industrial interest during the last two decades owing to their exceptional physical and chemical properties $[1,2]$. These nanostructured carbon materials belong to a new class of support for use in several catalytic domains, i.e. gas- and liquid-phase processes, with catalytic performances often higher than that encountered over traditional grain-shaped catalysts.

Large scale syntheses of carbon nanostructures, nanotubes and nanofibres, are today mainly conducted through a catalytic route due to the high yield, high selectivity and low cost $[3,4]$, when compared to the traditional physical routes such as laser ablation or arc-discharge [5-9]. Moreover, the control of the nanostructure morphology can be accurately realized through the tuning of the reaction parameters and the catalyst composition. The high selectivity of the catalytic method towards the formation of carbon nanostructures also avoids the need for a post-purification step which is a time and cost-consuming process. Despite the significant amount of research which has been initiated on this subject, the large scale synthesis of nanostructured materials with high purity for potential applications is still being investigated. In three recent studies, the reaction and synthesis parameters of carbon nanotubes, relatively high yields were reported [8-11]. A high yield of multi-walled carbon nanotubes by fluidized bed have also been reported by Corrias et al. [12].

The aim of the present work is to report the large scale, i.e. several grams per hour, synthesis of multi-walled carbon nanotubes (MWNTs) with a uniform diameter by the chemical vapour deposition (CVD) method over Fe supported on alumina at relatively low temperature, i.e. $640{ }^{\circ} \mathrm{C}$, using a synthesis mixture consisting of ethane and hydrogen in an appropriate ratio. Ethane was preferred as carbon source as because, already reported by our group, ethane allows the synthesis of carbon nanofibers with a significant yield, i.e. $50 \mathrm{~g}$ par gram of nickel per hour $[13,14]$. One of the highest reported in the open literature to date. Ethane is also a cheap and non-toxic carbon source compared to other gaseous carbon sources. The quality and the microstructure of the carbon nanotubes were investigated by scanning electron and transmission electron microscopy. 


\section{Experimental}

The support used was a high surface area $\gamma-\mathrm{Al}_{2} \mathrm{O}_{3}(\mathrm{CK}$ 300B Ketjen with a surface area of $220 \mathrm{~m}^{2} \cdot \mathrm{g}^{-1}$ ) which was mainly made up of a mesoporous network. The alumina support was crushed and sieved and a fraction of 80-150 $\mu \mathrm{m}$ was retained for catalyst preparation. The catalyst was prepared using an aqueous solution of $\mathrm{Fe}\left(\mathrm{Fe}\left(\mathrm{NO}_{3}\right)_{3} \cdot 9 \mathrm{H}_{2} \mathrm{O}\right)$, with the Fe concentration fixed at $20 \mathrm{wt} . \%$. The wet solid was dried at $100{ }^{\circ} \mathrm{C}$ and then calcined in air at $450{ }^{\circ} \mathrm{C}$ for $2 \mathrm{~h}$ in order to obtain the oxidic form of the catalyst precursor. The catalyst $(0.06 \mathrm{~g})$, in its oxidic form, was placed in a ceramic boat inside a quartz tube (diameter, $30 \mathrm{~mm}$, length, $600 \mathrm{~mm}$ ), located in an horizontal electrical furnace, and flushed for 30 minutes in flowing hydrogen at room temperature and finally reduced at $650{ }^{\circ} \mathrm{C}$ for $2 \mathrm{~h}$. After this treatment the hydrogen flow was replaced by a mixture of ethane and hydrogen $\left(60: 60 \mathrm{ml} . \mathrm{min}^{-1}\right)$ and the temperature of the furnace was rapidly increased $\left(20^{\circ} \mathrm{C} \cdot \mathrm{min}^{-1}\right)$ to $750{ }^{\circ} \mathrm{C}$. The synthesis was carried out at $750^{\circ} \mathrm{C}$ for $2 \mathrm{~h}$. After synthesis the raw products were treated with a solution of $\mathrm{KOH}(1 \mathrm{M})$ at $80{ }^{\circ} \mathrm{C}$ in order to remove the alumina support. The resulting material was washed several times with distilled water and then treated with a solution of $\mathrm{HCl}(1 \mathrm{M})$ in order to remove the remaining iron particles, it was then washed with distilled water before being dried at $200^{\circ} \mathrm{C}$.

Carbon yields were calculated by weighing the sample after the reduction process and after the carbon nanotube synthesis according to the following formula:

$$
\text { CNTs yield }(\text { wt. } \%)=\left[\left(M_{F}-M_{I}\right) / M_{I}\right] \times 100
$$

where $M_{F}$ is the total weight obtained after synthesis, $M_{I}$ is the weight of the catalyst after reduction.

X-ray diffraction (XRD) patterns of powdered samples were recorded at room temperature using a Siemens D-5000 diffractometer with a $\mathrm{Cu}-\mathrm{K} \alpha$ non-monochromatic radiation source $(\lambda=1.54056 \AA)$. Scanning electron microscopy (SEM) was performed on a JEOL $6700 \mathrm{~F}$ microscope with an accelerated voltage of $3 \mathrm{kV}$. Transmission electron microscopy (TEM) was carried out on a Topcon 002B-UHR microscope working under an accelerated voltage of $200 \mathrm{kV}$ and with a point-to-point resolution of $0.17 \mathrm{~nm}$. Surface area was measured using a Coulter SA-3100 sorptometer with $\mathrm{N}_{2}$ as adsorbant at liquid nitrogen 
temperature. Before measurement the samples were outgased at $200{ }^{\circ} \mathrm{C}$ for $2 \mathrm{~h}$ in order to desorb moisture and other weakly adsorbed residues.

\section{Results and discussion}

\subsection{SEM characterisation}

The morphology of the as-synthesized carbon nanostructures was investigated by SEM. A SEM image (Figure 1A) shows the presence of a homogeneous distribution of carbon nanotubes covering the alumina-based catalyst surface in a web-like network. High-resolution SEM images of the purified sample shows that the carbon nanotubes were homogeneous in diameter, ca. $40 \mathrm{~nm}$, and with lengths up to micrometers (Figure 1B) resulting in an average aspect ratio $>50$.
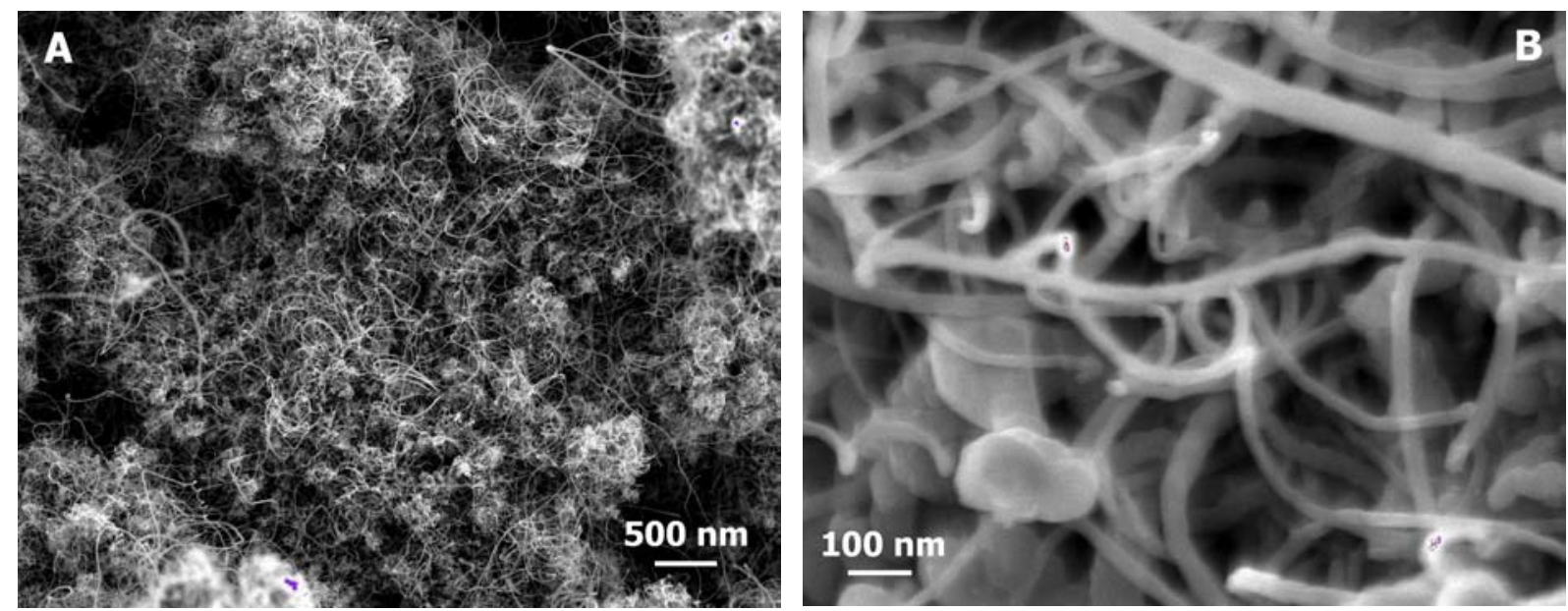

Fig. 1. SEM images of the MWNTs synthesized in flowing $\mathrm{C}_{2} \mathrm{H}_{6}$ and $\mathrm{H}_{2}$ over $\mathrm{Fe} / \gamma-\mathrm{Al}_{2} \mathrm{O}_{3}$ catalyst at $750{ }^{\circ} \mathrm{C}$ after post-synthesis purification process in order to remove the catalyst. (A) Low-magnification SEM observation of the raw material, i.e. MWNTs and catalyst. (B) Highmagnification SEM image of the purified MWNTs showing the high selectivity towards carbon nanotubes with homogeneous diameter.

Statistical SEM observation revealed that the selectivity towards carbon nanotubes was extremely high and no traces of onion-like or carbon particles were observed in the sample. The selective growth of carbon nanotubes requires a perfect balance between the rate 
of carbon supply and that of solid carbon precipitation otherwise amorphous carbon can buildup on the catalyst surface leading to deactivation and the formation of impurities.

The carbon nanotubes obtained by the CVD method are less straight than those obtained by physical methods such as arc discharge or laser ablation. This is attributed to the low temperature synthesis used in the CVD method which favours the formation of structural defects responsible for the bending or twisting of the tubes. However, a high synthesis temperature, i.e. $800{ }^{\circ} \mathrm{C}$ instead of $750{ }^{\circ} \mathrm{C}$, keeping other reaction conditions similar led to the formation of carbon particles instead of carbon nanotubes as shown in Figure 2 where a mixture of nanotubes and nanoparticles was observed. It is expected that at high reaction temperature part of the carbon gaseous source was directly underwent pyrolysis leading to the formation of carbon nanoparticles or turbostratic structure instead to be transformed into carbon nanotubes.

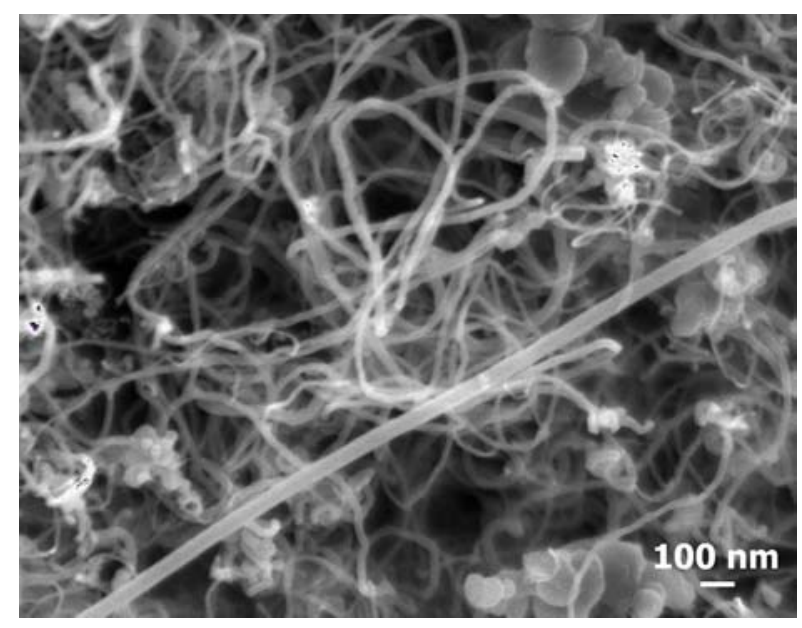

Fig. 2. SEM image of a mixture of carbon nanotubes and carbon nanoparticles synthesized at $800{ }^{\circ} \mathrm{C}$ in the presence of $\mathrm{C}_{2} \mathrm{H}_{6}$ and $\mathrm{H}_{2}$ mixture. The high temperature of synthesis also leads to the formation of MWNT with different diameter.

\subsection{TEM characterisation}

The low magnification TEM image (Figure 3A) shows that the carbon nanotubes were the only product obtained and no trace of carbon nanoparticles (less than 1 to $2 \%$ according to the statistical TEM observation, was observed in the sample, which indicated the high selectivity of the synthesis method in good agreement with the SEM results. TEM observation 
also showed that some tubes were bent where defects occured during the growth, leading to a change in the growth direction. The carbon nanostructures were multi-walled carbon nanotubes (MWNTs) with diameters ranging from 15 to about $50 \mathrm{~nm}$ and lengths up to several hundred nanometers leading to an aspect ratio $>50$. High-resolution TEM image shows that the nanotubes were made of parallel graphene planes oriented along the tube axis and only a very small amount of amorphous carbon was observed on their outer surfaces (Figure 3B). The amorphous carbon originated from the pyrolysis of the hydrocarbon during the cooling of the system; this is frequently reported in the CVD synthesis [9]. This phenomenon could be avoided or diminished by rapid evacuation of the reaction atmosphere at the end of the synthesis as reported by Emmenegger et al. [9]. The interplanar distance between two adjacent graphene planes was about $0.34 \mathrm{~nm}$ which is close to that of the [002] interplanar distance in graphite, i.e. $0.335 \mathrm{~nm}$. The higher interplanar distance observed on the MWNTs was attributed to the curved shape of their structures which induce strain inside the graphene planes stacking [1]. Due to the low reaction temperature some defects could be observed between the graphene planes (indicated by arrows in the Figure 3A).
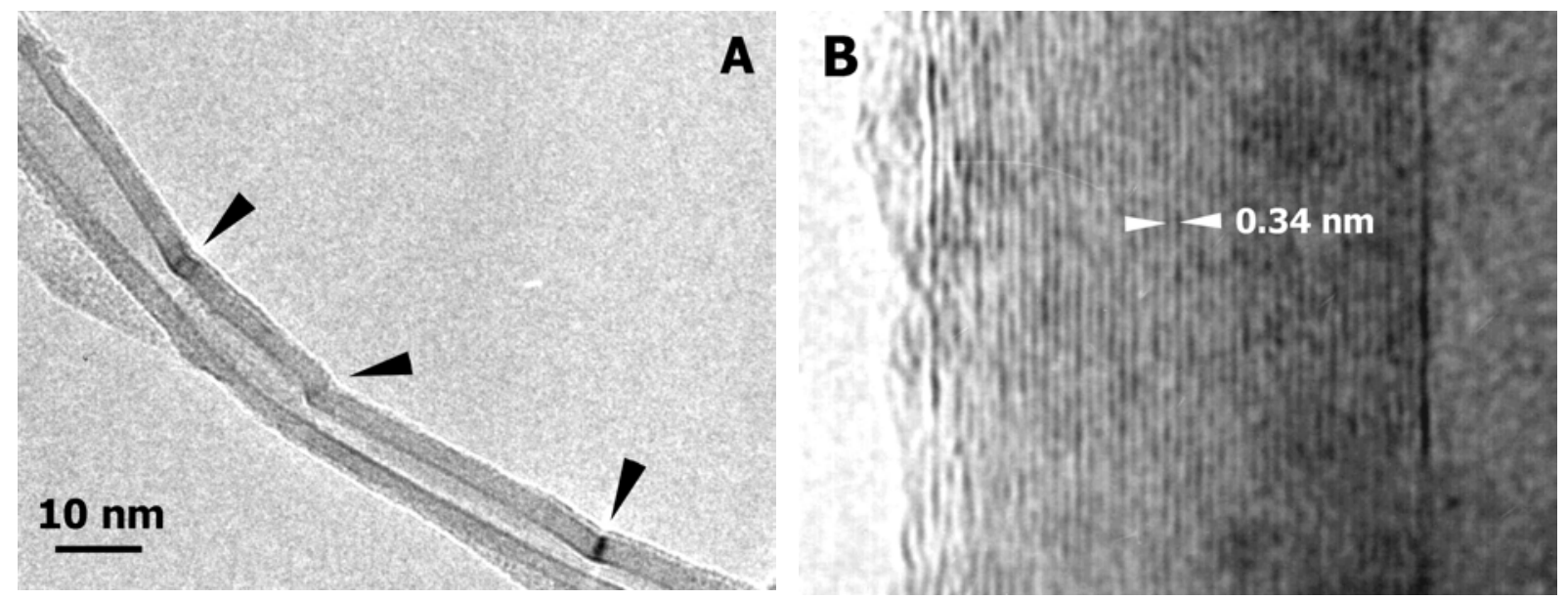

Fig. 3. TEM images of the MWNTs with different magnifications. The low-magnification image (A) reveals the presence of some structural defects along the tube axis (arrow). The high-resolution image (B) clearly shows the stacking of the graphene basal planes parallel to the tube axis. The graphene planes were not perfectly parallel and some defects can be observed (indicated by arrows).

The bamboo-like or nanoparticles of carbon were completely absent from the sample and only tubes, more or less straight, were observed. This could be attributed to the interactions 
between the deposited iron phase and the support. Ermakova et al. [16] have reported that during the synthesis of MWNTs over iron supported on $\mathrm{TiO}_{2}$ several types of carbon nanofilaments, i.e. straight, bamboo-like or nanoparticles, were observed. On the other hand, when $\mathrm{TiO}_{2}$ was replaced by $\mathrm{SiO}_{2}$ a smaller number of bamboo-like carbon structure was observed. The interaction between the active phase and the support explain these results.

Careful high-magnification TEM revealed the presence of nanotubes both with an open and with a closed tips (Figure 4). No iron metal particles were observed on the tube tips indicating that the main growth mode followed a base growth mechanism [17]. Some iron particles were observed inside the carbon nanotube bodies (arrow in Fig. 4) which indicates that during the growth process some iron was carried out of the support surface by the tube growth.

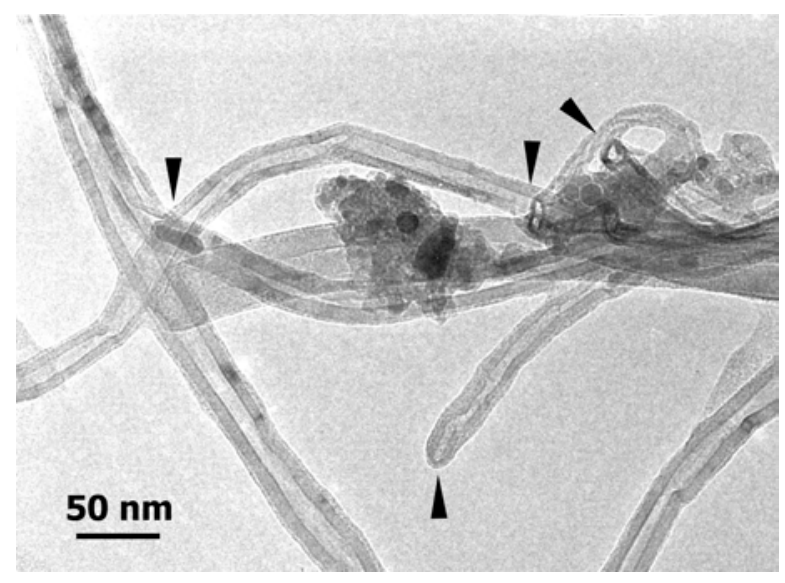

Fig. 4. TEM image of MWNTs synthesized by the CVD method with open and closed tube tips. The presence of some encapsulated iron inside the tubes was also observed.

It is thought that during the carbon diffusion process through the iron lattice an elevation of temperature occured causing oscillation of the lattice and a rise in temperature. As a result, the iron was transformed from the solid state into a quasi-liquid state despite the fact that the synthesis temperature was far lower than the melting temperature of iron. The quasi-liquid iron phase was extracted during the MWNT growth and, remained in a encapsulated iron phase within the carbon nanotube channel and thus, was unaccessible for the further MWNT growth resulting to a gradual loss of the MWNT production with time on stream. A similar mechanism of MWNT growth over iron-based catalyst has been proposed 
by Ermakova et al. [16]. This encapsulation process provides an explanation why the yield of carbon nanotubes was significantly lower than the yield of carbon nanofibers over a nickel catalyst where almost no fragmentation was ever observed. Park and Keane [18] have recently reported that, during the growth of carbon nanofibers from a nickel catalyst some nickel fragmentation was also observed. However, this phenomenon was observed with a significant lesser extent than that seen with the iron phase.

\subsection{Specific surface area measurements}

The specific surface area of the MWNTs after the purification steps was between 100 and $160 \mathrm{~m}^{2} \cdot \mathrm{g}^{-1}$ depending on the synthesis conditions. This was close to the data reported in the literature as in general the surface area of the MWNTs determined by BET techniques is 20 to $100 \mathrm{~m}^{2} . \mathrm{g}^{-1}$ ranging between the surface area of graphite and of activated porous carbons [1]. It is worth noting that the measured specific surface area was mainly linked with a mesoporous network as no trace of micropore was observed among the sample.

\subsection{MWNT yield as a function of the synthesis duration}

According to the calculation already described a carbon nanotube yield of $20 \mathrm{~g}$ of carbon nanotubes per gram of catalyst, was obtained over the $\mathrm{Fe}-20 \mathrm{wt} . \% / \mathrm{Al}_{2} \mathrm{O}_{3}$ catalyst at temperature ranging from 650 to $720^{\circ} \mathrm{C}$. Optical micrographs of the starting catalyst and the final product are presented in Figure 5 to illustrate the possibility to synthesize MWNT with high purity and yield. Work is ongoing to check the influence of the synthesis conditions, i.e. temperature, flow rate and duration, and the concentration of the gaseous carbon source on the total MWNT yield. 

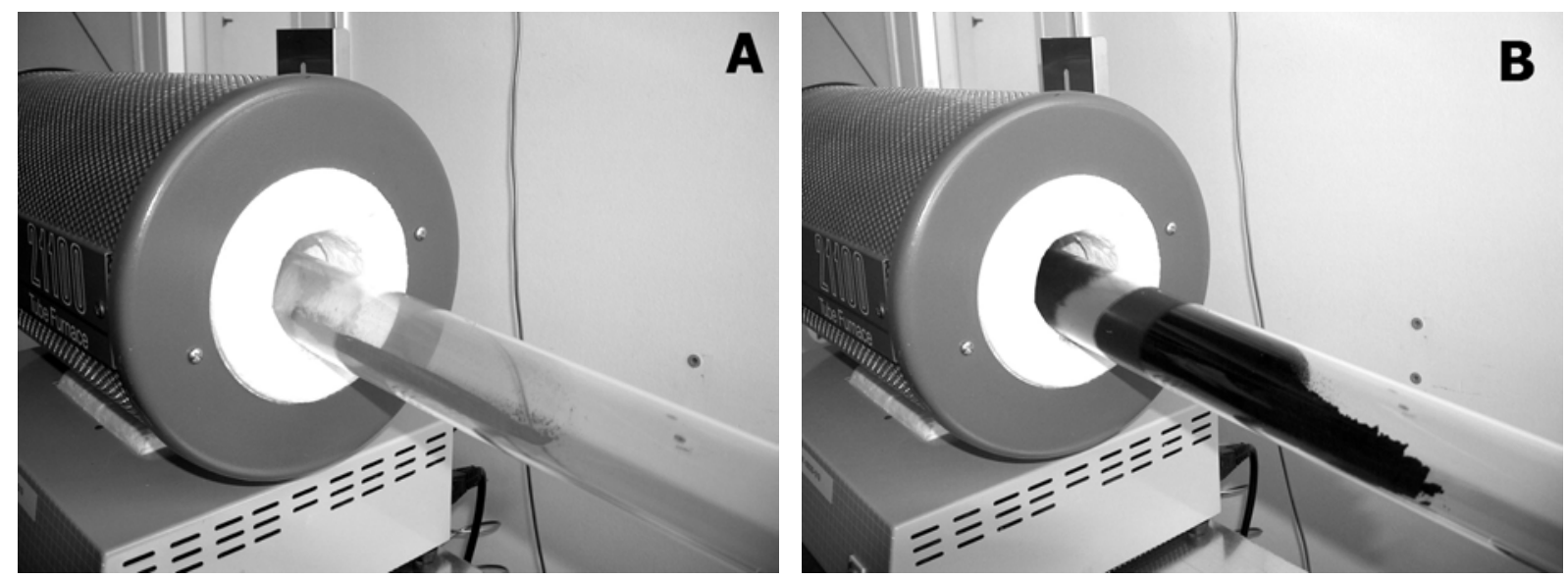

Fig. 5. Experimental set-up for carbon nanotubes synthesis : (A) $\mathrm{Fe} / \mathrm{Al}_{2} \mathrm{O}_{3}$ catalysts before MWNTs synthesis and (B) MWNTs produced after synthesis

The nucleation of filamentous carbon requires a supersaturation of carbon in iron, followed by a phase transformation of the starting iron into $\mathrm{Fe}_{3} \mathrm{C}$ and precipitation of carbon layers at the metal-support interface to yield carbon nanotubes. According to the proposed mechanism the nature of the gaseous hydrocarbon plays an important role in the final yield: the degree of supersaturation is generally determined by the affinity for carbon formation of the gas phase. The nature of the metal-support interface also strongly influences the rejection or precipitation of carbon at this interface. Iron supported on silica catalyst was more active for carbon nanotube formation than iron supported on silicon carbide despite the similarity in the precursor phase nature. The carbon nanotube growth and selectivity were also strongly governed by the reaction temperature for a given reactant mixture and a given catalyst. In the presence of a mixture of ethane and hydrogen the optimal synthesis temperature for high quality carbon nanotubes was around $750{ }^{\circ} \mathrm{C}$. At a higher temperature, i.e. $800{ }^{\circ} \mathrm{C}$, carbon nanotubes of lower quality were obtained, i.e. nanotubes and amorphous carbon (not shown). Apparently, at high reaction temperature the rate of hydrocarbon decomposition and amorphous carbon formation were high enough to overcome that of the MWNT growth.

It was expected that when $\mathrm{C}_{2} \mathrm{H}_{6}$ was used as carbon source the number of carbon nuclei formed on the catalyst surface would significantly increase compared to those formed on silica or carbon supports, which in turn would accelerate the rate of carbon diffusion from the carbide surface to the carbide-support interface where carbon nanotubes were precipited. 
The solubility of carbon in the iron carbide surface significantly differed from the equilibrium value at the carbide-support interface and therefore a driving force for the diffusion of carbon through the iron carbide particles was initiated, leading to continuous growth of the carbon nanotubes with the concomitant formation of $\alpha-\mathrm{Fe}$ which allowed further decomposition of the hydrocarbon to yield carbon for subsequent tube growth. Yang et al. [19] have reported that the carbon concentration near the nickel particles in contact with a mixture of methane and hydrogen was $35 \%$ higher than for a mixture in equilibrium with graphite.

\section{Conclusion}

In summary, $\mathrm{C}_{2} \mathrm{H}_{6}$ seems to be an appropriate carbon gaseous source for growing multi-walled carbon nanotubes selectively and with a high yield using the CVD method at the relatively low temperature of $650-750{ }^{\circ} \mathrm{C}$. Hydrogen also plays an important role in the synthesis by preventing the catalytic site from being encapsulated by graphite and consequently avoiding deactivation during syntheses of long duration. The selectivity of the carbon nanotubes synthesized in the present work was high, avoiding the need for a costly purification step to eliminate by-products, as generally encountered with physical methods such as arc-discharge or laser ablation. According to the present synthesis several grams of pure MWNTs with homogeneous diameters of about $20 \mathrm{~nm}$ were obtained on the basis of $1 \mathrm{~g}$ of catalyst after $4 \mathrm{~h}$ of reaction at $750{ }^{\circ} \mathrm{C}$.

The possibility to synthesize carbon nanotubes with high selectivity and quality in mild conditions makes them more appealing for use in other applications where the exceptional physical and chemical properties of these materials would be of great benefit. Work is on-going to evaluate the catalytic properties of these nanomaterials in several gas and liquid-phase reactions, and also to study the influence of the nature of the active phase and the support on the final carbon nanotubes yield.

\section{Acknowledgements}

GG would like to thank the Italian Ministry of University and Scientific Research for the grant during his research stay in LMSPC (France). RV would like to thank the CNPq (Brazil) and Sicat SA for financial support. S. Julie (IPCMS) is gratefully acknowledged for TEM analysis. 


\section{References}

[1] Ajayan, P. M. Chem. Rev. 99 (1999) 1797.

[2] H. J. Dai, Acc. Chem. Res. 35 (2002) 1045.

[3] M. Pérez-Cabero, I. Rodriguez-Ramos, A. Guerrero-Ruiz, J. Catal. (2003) in press.

[4] S. Tang, Z. Zhong, Z. Xiong, L. Sun, L. Liu, J. Lin, Z. X. Shen, K. L. Tan, Chem. Phys. Lett. 350 (2001) 19.

[5] X. Zhao, M. Ohkohchi, H. Shimoyama, Y. Ando, J. Crystal Growth 198 (1999) 934.

[6] J. M. Bonard, L. Forro, D. Ugarte, W. A. de Heer, A. Châtelain, ECC Research 1 (1998) 9.

[7] T. W. Ebbesen, P. M. Ayajan, Nature 358 (1992) 220.

[8] Ph. Mauron, C. Emmenegger, A. Züttel, C. Nützenadel, P. Sudan, L. Schalapbach, Carbon 40 (2002) 1685.

[9] C. Emmenegger, J-M Bonard, P Mauron, P. Sudan, A. Lepora, B Grobety, A Zuttler L. Sschapbach, Carbon 41 (2003) 539.

[10] P Mauron C. Emmenegger, P. Sudan, P. Wagner, S. Rentsch, A. Zuttler, Diam. Rel. Mater. 12 (2003) 780.

[11] N. Nagaraju, A. Fonseca, Z. Konya, J.B. Nagy, J. Mol. Catal. A: Chem. 181 (2002) 57.

[12] M. Corrias, B. Caussat, A. Ayral, J. Durand, Y. Kihn, Ph. Kalck, Ph. Serp, Chem. Eng. Sci. 58 (2003) 4475.

[13] R. Vieira, C. Pham-Huu, N. Keller, M. J. Ledoux, Chem. Commun. (2002) 954.

[14] C. Pham-Huu, N. Keller, V. V. Roddatis, G. Mestl, R. Schlögl, M. J. Ledoux, Phys. Chem. Chem. Phys. 4 (2002) 514.

[15] P. S. Pilipenko, V. V. Veselov, Poroshk. Metall. 150 (1975) 9.

[16] M. A. Ermakova, D. Y. Ermakov, A. L. Chuvilin, G. G. Kuvshinov, J. Catal. 201 (2001) 183.

[17] C. Laurent, E. Flahaut, A. Peigney, A. Rousset, New J. Chem. 1(1998) 229 and references therein.

[18] C. Park, M. A. Keane, J. Catal. 221 (2004) 386.

[19] R. T. Yang, P. J. Goethel, J. M. Schwartz, C. R. F. Lund, J. Catal. 122 (1990) 206. 